\title{
VIBRATING WIRE FIELD-MEASURING TECHNIQUE *
}

\author{
Alexander Temnykh ${ }^{\dagger}$ \\ Laboratory of Nuclear Studies, Cornell University, Ithaca NY, USA
}

The Vibrating wire field-measuring technique presented here is dedicated to the problem of alignment of quadrupole magnets and is based on the following principle. The stretched wire has vibrational modes consisting of the fundamental mode and higher harmonics. The half wave length of the fundamental mode is equal to the length of the wire. Suppose there is a transverse magnetic field surrounding wire. If the frequency of the current in the wire is an eigenmode frequency of wire vibration, it will excite a corresponding harmonic. The strength and phase of excitation will depend on the field distribution along the wire. Using various frequencies and measuring amplitudes and phases of the resulting vibrations, one can extract information about the field distribution in order to reconstruct it. The field in turn shows the misalignment of quadrupoles.

In comparison with the pulsed-wire method, see [1][4], the vibrating wire technique does not require the wire length to be longer than the length of the test region, and due to its extraordinary sensitivity it does not require the higher voltage for the long wire scheme. Therefore, it may be more appropriate for some projects.

\section{THEORY}

Figure 1 displays the setup used in experiments and in calculations below. A wire with tension $T$ and length $l$ has the fixed ends at $x=0$ and $x=l$. The current, $\mathrm{I}(\mathrm{t})$, in the wire, i.e., driving current, depends on time as $I(t)=I_{0} \exp (i \omega t)$. The wire crosses a region with horizontal magnetic field, $B_{y}(x)$, which is zero at the ends, $B_{y}(0)=B_{y}(l)=0$. Consider the vertical plane. There are two forces affecting the wire. They are gravity $g \cdot \mu$, where $\mu$ is mass of wire per unit of length, and the Lorentz force $B_{y}(x) \cdot I(t)$.

The equation for vertical wire position, $U(x, t)$, will be:

$$
\mu \frac{\partial^{2} U}{\partial t^{2}}=T \frac{\partial^{2} U}{\partial x^{2}}-\gamma \frac{\partial U}{\partial t}-\mu g+B_{y}(x) \cdot I_{0} \exp (i \omega t)
$$

With boundary condition:

$$
U(t, 0)=U(t, l)=0
$$

Here term $\gamma \frac{\partial U}{\partial t}$ describes damping. A general solution may be written in the form:

$$
\begin{aligned}
U(x, t) & =U_{g}(x)+U_{d}(x, t) \\
U_{d}(x, t) & =U_{b}(x) \cdot \exp (i \omega t)
\end{aligned}
$$

Where $U_{g}(x)$ is the gravity term and $U_{d}(x, t)$ is the dynamic term caused by the interaction between the magnetic field and the driving current. An approximate expression for $U_{g}(x)$ is:

\footnotetext{
* Work supported by the National Science Foundation
}

$\dagger$ On leave from BINP, Novisibirsk

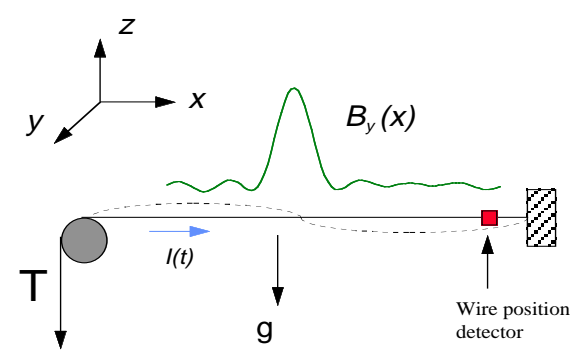

Figure 1: Scheme of vibrating wire experiments.

$$
U_{g}(x)=-\frac{\mu g}{2 T} x(x-l)
$$

Note that at the point $x=l / 2$ function $U_{g}(x)$ reaches its minimum which is the sag, $\mathrm{S}$.

$$
S=-\frac{\mu g}{8 T} l^{2}
$$

Function $U_{b}(x)$, determined by magnetic field, may be found in the following way. As $U_{b}(x)$ satisfies the condition $U_{b}(0)=U_{b}(l)=0$, it can be represented by Fourier sine series:

$$
U_{b}(x)=\sum_{n=1}^{\infty} U_{n} \sin \left(\frac{\pi n}{l} x\right)
$$

The magnetic field $B_{y}(x)$, with similar boundary conditions, may be represented in the same way:

$$
B_{y}(x)=\sum_{n=1}^{\infty} B_{n} \sin \left(\frac{\pi n}{l} x\right)
$$

Substituting 3,4,6 and 7 into equation 1 we set:

$$
\begin{aligned}
\sum_{n=1}^{\infty} U_{n} \cdot\left(\omega^{2}-\omega_{n}^{2}\right. & +i \gamma \omega) \sin \left(\frac{\pi n}{l} x\right) \\
& =\sum_{n=1}^{\infty} \frac{I_{0} B_{n}}{\mu} \sin \left(\frac{\pi n}{l} x\right) \\
\omega_{n} & =2 \pi \frac{n}{2 l} \sqrt{\frac{T}{\mu}}
\end{aligned}
$$

Combining expression 9 with the formula for sag, (see 5), one can find a simple relation between sag and the fundamental mode frequency: 


$$
S=\frac{g}{32}\left(\frac{2 \pi}{\omega_{1}}\right)^{2}
$$

This means that to obtain the sag we only need to measure the fundamental frequency and using $g=$ $9.80665 \mathrm{~m} / \mathrm{sec}^{2}$ we can precisely calculate the sag.

By comparing the right and left sides of equation 8 one can find a connection between coefficients $U_{n}$ and $B_{n}$ and write the dynamic part of the general solution of equation 1 as:

$$
U_{d}(x, t)=\sum_{n=1}^{\infty} \frac{B_{n} \sin \left(\frac{\pi n}{l} x\right)}{\left(\omega^{2}-\omega_{n}^{2}+i \gamma \omega\right)} I_{0} \exp (i \omega t)
$$

Consider how to obtain coefficients $B_{n}$ of expansion 7 and reconstruct the magnetic field knowing the wire motion at the sensor location. Suppose the wire position sensor is located at the point $x=x_{s}$. Let us construct the function $\mathcal{F}(\omega)$ which is the time average of the product of wire position, $U_{d}\left(x_{s}, t\right)$, and driving current, $I(t)$ :

$$
\begin{gathered}
\mathcal{F}(\omega)=\frac{1}{T} \int_{0}^{T} U_{d}\left(x_{s}, t\right) I(t) d t=\sum_{n=1}^{\infty} \mathcal{F}_{n}(\omega) \\
\mathcal{F}_{n}(\omega)=\frac{B_{n} I_{0}^{2}}{2 \mu} \sin \left(\frac{\pi n}{l} x_{s}\right) \frac{\left(\omega-\omega_{n}\right)}{4 \omega\left(\omega-\omega_{n}\right)^{2}+\omega \gamma^{2}}
\end{gathered}
$$

Note that due to weak damping the wire motion has a high quality factor $Q$. It causes strong resonance amplification, and if the frequency of driving current is near $\omega_{n}$, i.e., $\omega \approx \omega_{n}$, the resonance term $\mathcal{F}_{n}$ in equation 12 will dominate over the rest, i.e. , $\mathcal{F}(\omega) \approx \mathcal{F}_{n}(\omega)$. Suppose we scan the driving current frequency through one of resonance frequencies, $\omega_{n}$. By recording both the driving current and signal from wire position sensor and doing numerical integration, (see formula 12), we can measure function $\mathcal{F}_{n}(\omega)$. Then we have to fit this measurement with the formula:

$$
\mathcal{F}_{n}(\omega)=a_{n} \frac{\left(\omega-b_{n}\right)}{4 \omega\left(\omega-b_{n}\right)^{2}+\omega c_{n}^{2}}
$$

where $a_{n}, b_{n}, c_{n}$ are free parameters. Component $B_{n}$ is obtained from $a_{n}$ as:

$$
B_{n}=a_{n} \frac{1}{\sin \left(\frac{\pi n}{l} x_{s}\right)} \frac{2 \mu}{I_{0}^{2}}
$$

Repeating this procedure for various $\omega_{n}$, and using equation 7, we will reconstruct the magnetic field profile along the wire. Note that the resolution of reconstruction will depend on how many harmonics will be used and will be approximately equal to the shortest wavelength of the excited modes. Motion in the horizontal plane does not differ from vertical motion except for the gravity term. The next sections describe model measurements and the practical application of the vibrating wire technique.
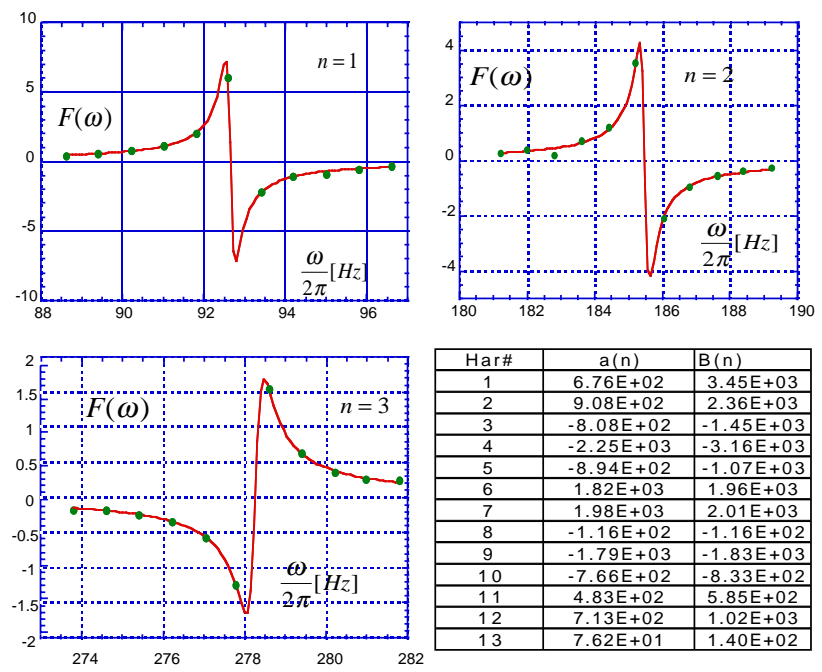

Figure 2: Measured function $\mathcal{F}(\omega)$ and coefficients $a_{n}$ obtained from fitting. Points show measurements, lines are fits with formula $\mathcal{F}_{n}(\omega)=a_{n} \frac{\left(\omega-b_{n}\right)}{4 \omega\left(\omega-b_{n}\right)^{2}+\omega c_{n}^{2}}$

\section{MEASUREMENT}

Wire used in the experiments was $100 \mu \mathrm{m}$ diameter made with alloy of copper-beryllium. A phototransistor-LED assembly, (Motorola H21A1), was deployed as a wire position monitor. This kind of device was widely used in previous work, (see for example [2] and [4]). Note that the assembly may be oriented in such way that it will effectively sense wire motion in one plane, in horizontal or in vertical . To detect motion in other plane the assembly must be rotated by 90 degrees. Macintosh Quadra 800 with LabNB Interface Board provided almost all needs of the measurements. An application program created in LabVIEW scanned the driving current frequency, recorded and manipulated signals, made fits and etc. The final output yields the magnetic field measured along wire.

A one meter long wire was used in the first modeling measurement. The wire motion detector was placed $7.5 \mathrm{~cm}$ from one of the ends. A small permanent magnet placed along the wire created a magnetic field for testing. The strength of the field was controlled by the distance between magnet and wire.

Figures 2 and 3 illustrate the description given in the previous section. Plots in figure 2 show the results of excitation of the first three harmonics out of thirteen which were used in the measurement. Points show the measured function $\mathcal{F}(\omega)$ defined in the theoretical section. A line is fit to formula 1. There is a good agreement between the measurement and fitting in all cases. The table on figure 2 shows the parameters $a_{n}$ obtained from fitting all 13 harmonics. and coefficients $B_{n}$ calculated from $a_{n}$. The field distribution reconstructed by equation 7 is plotted in figure 3 . It shows a very clear signal from the magnetic field. Note that the width of the signal, $\sim 10 \mathrm{~cm}$, is determined by the shortest harmonic wavelength used in the measurement.

The next experiment was to find the magnetic center of permanent magnet quad using a longer wire. Available setup space allowed use of wire of $3 m$ length. The test 


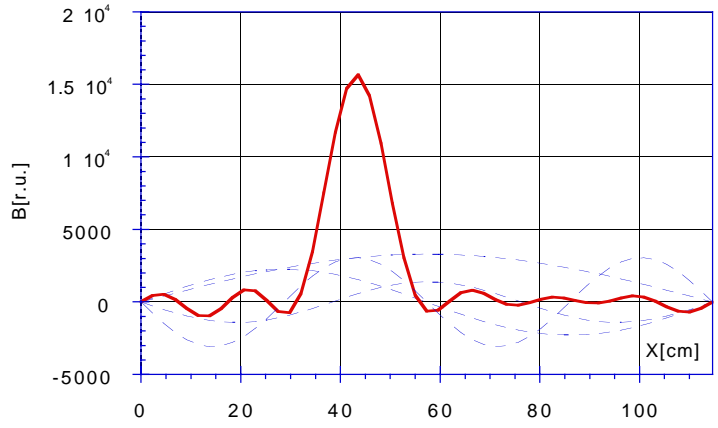

Figure 3: Components $B_{n}$, (see plot a), calculated from coefficients $a_{n}$ and reconstructed magnetic field, (see plot b). Dotted lines show the first 5 harmonics corresponding to $B_{n}$.

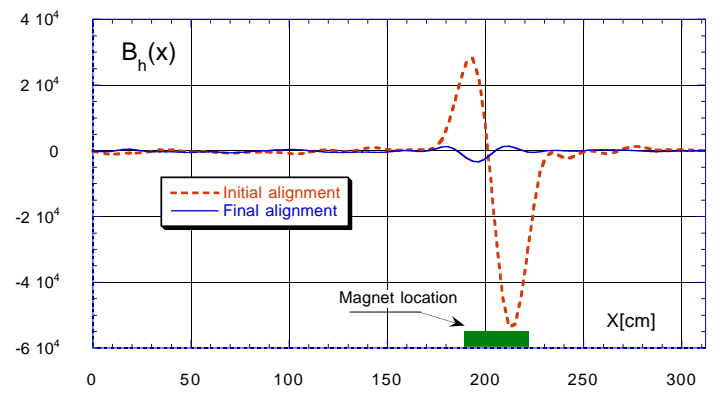

Figure 4: Horizontal magnetic field along wire after conventional alignment, (see dotted line), and after vertical alignment with vibrating wire technique, (see solid line).

permanent magnet quad was $30 \mathrm{~cm}$ long with a $14.8 \mathrm{~T} / \mathrm{m}$ gradient.

In the beginning, the mechanical center of quad was adjusted to the wire position with traditional measuring tools, i.e., using rulers. The precision of this alignment was approximately $0.1 \mathrm{~mm}$. Note that magnetic center of the quad may differ from its mechanical center.

The vibrating wire measurement gave the horizontal field distribution shown by the dotted line in figure 4 . The square at the bottom shows the location and size of the test magnet. In the measurement 30 harmonics were used, with shortest wavelength equal to $20 \mathrm{~cm}$. This wavelength is 1.5 times shorter than the length of the test magnet and allowed us to see details of the field generated by magnet. This plot shows two unequal maxima of different polarities which correspond to the magnet ends. This kind of field distribution is due to a combination of tilt and shift of magnetic axes in the vertical plane relative to the wire. The solid line on figure 4 shows the final field distribution after a few iterations of measurement and adjustment of vertical quad position. The maximum amplitude is approximately 10 times less than it was in the beginning.

After the quad's magnetic center was adjusted to the wire position in the vertical and horizontal plane, using high precision optical instruments it was found that the misalignment between geometrical and magnetic center was in range between $125 \mu \mathrm{m}$ and $500 \mu \mathrm{m}$.

\section{DISCUSSION AND CONCLUSION}

Consider the time needed for measurement. The weak damping of wire vibration which gives the enormous sensitivity of the technique slows the process of measurement. After each frequency change one must wait for about 35 seconds to reach equilibrium of wire motion and then record signals. Due to this delay the measurement consisting of many harmonics may take a relatively long time. For example, the time for measurement consisting of 13 harmonics with 11 steps of frequency scan for each of them, (see figure 3), was about 10 minutes. Another example, (figure 4), consisting of the analysis of 30 harmonics required 25 minutes. However the magnet alignment described above, did not require analysis of so many harmonics. Only two of them, most sensitive to the magnet shift and tilt, were used during this procedure. The full measurements, i.e. using 30 harmonics, were done just in the beginning and at the end to verify final result.

The another aspect is the effect of non-zero field at one of the ends of wire. One can suspect that this field may have propagated an effect along wire. This arrangement has been simulated with software "Mathematica" for Macintosh and two important conclusions have been derived. The difference between "real" and "measured" field profiles is localized around the wire end in a length of region approximately equal to that of the shortest wave length and a non-zero field at the wire end does not have an effect on the determination of the field profile away from this end.

Theoretical consideration and experiments reported above demonstrate that the vibrating wire technique is a convenient method for the precise alignment of magnetic centers of a series magnetic elements. It has enormous sensitivity and does not require any special equipment.

In comparison with the pulsed-wire technique it does not need more space than the length of the test region and does not require generating very high voltage pulses on a long wire.

\section{ACKNOWLEDGMENT}

I thank James Welch, who motivated me to study the problem of magnet alignment. I am also very grateful to Boyce McDaniel, David Rice, David Rubin and Scott Chapman for help and to Alexander Mikhailichenko for useful comments.

\section{REFERENCES}

[1] R. Warren and C. Elliot, New System for Wiggler Fabrication and Testing, Los Alamos National Laboratory, LA-UR 87-2981, (1987)

[2] R.W. Warren, NIM A272 (1988) 257-263

[3] C.M. Fortgang, et.al., Pulsed Taut-Wire Alignment of Multiple Permanent Magnet Quadrupoles, 1990 LINAC Conference, Albuquerque, NM, Sept. 1990.

[4] J.G. Melton, et.al., Pulsed Taut-Wire Measurement of Magnetic Alignment of the ITS Induction Cells, in Proceedings of the 1993 IEEE Particle Accelerator Conference, Catalog No. 0-7803-1203-1/9303.00, p. 2944 